\title{
Light Prestressed Segmented Arch (LPSA) Bridges: A Demonstration of Sustainable Engineering
}

Ibrahim Al-Khattat, Ph.D., MIMechE, SustainableScience.org Inc., Iowa, USA

\begin{abstract}
Summary
A jointing method combining slide-fitting with tension-induced compression is presented. It eliminates the "analysis black box" at each node of a timber structure. Mathematical and computational modeling is further enhanced by the use of small diameter timber (SDT), due to its more predictable natural growthring structure. This is the core concept of the LPSA (light prestressed segmented arch) structural system as applied to timber-in-the-round. It represents a significant leap forward in the sustainable use of timber in construction, offering a sustainable alternative to steel and concrete, especially for bridges. The slidefitting jointing mechanism produces a unique property of harmless distortion energy dissipation via internal rigid-body motion. Therefore, LPSA structures have inherent resistance to the actions of earthquakes, hurricanes and flooding. The LPSA structural system outlines a framework for an urgently needed research, design and innovation field of sustainable engineering, in a world on the verge of environmental instability. SDT is a vast, untapped, sustainable resource. Its use as a premium construction material could provide a recipe for saving what remains of the world's forests and for profitable sustainable development. Two 19,5 m LPSA bridges have recently been constructed, using black locust (Robinia pseudoacacia) SDT. This is a least desirable but decay-resistant species with twoto-three times the crushing strength of concrete.
\end{abstract}

Keywords: light prestressed segmented arch (LPSA) structural system; small diameter timber (SDT); sustainable science and engineering; timber structures; stress analysis; joints; bridges.

\section{Introduction}

Increasing worldwide awareness about environmental sustainability and conservation of energy and natural resources necessitates a search for sustainable alternatives to the two major bridge construction materials. Problems with existing bridge technologies have recently been brought into focus by the 2007 Minnesota bridge collapse in the USA. Concrete and steel bridges are prohibitively expensive to build, maintain and repair, even for a rich country like the USA. At present, over a quarter of all US bridges need repair or replacement at an estimated cost of $\$ 63$ billions. $^{1}$

Commercial sawn timber is normally a bridge designer's distant third choice after steel and concrete, especially for important structures. Overall strength, durability and initial and maintenance costs are the main issues. However, the overriding fact against commercial sawn timber is this: although it comes from a renewable resource (mature trees), this resource is being consumed in a non-sustainable manner that also motivates destruction of the natural forests. Deforestation is one of the major sources of climate change and global warming.

It seems that an ideal bridge construction material for a "green" future would need to be environmentally sustainable, low in processing energy and overall costs and be "low-tech" to install, repair or replace. It must lead to aesthetic structures that would cost and weigh less, perform better and last longer than existing equivalents. It would be even more desirable if such structures were naturally resistant to earthquakes, hurricanes and flooding and if they could be dismantled for repair and reuse.

The purpose of this paper is to introduce a candidate for such a construction material, to present a candidate structural system that would utilize it most cost-effectively and efficiently and to point out the positive global implications of both environmentally, socially, economically and commercially.
The candidate material is a vastly abundant natural resource that has little or no commercial value at present. It is small diameter timber (SDT) with its natural growth-ring structure preserved for strength and predictability. Specifically for exposed structures like bridges, black locust, an invasive, decay-resistant hardwood species, ${ }^{2}$ is most suited.

The light prestressed segmented arch (LPSA) structural system resolves the chronic jointing problem that has so far inhibited large-scale profitable use of SDT as a premium structural material. For the first time, the LPSA connects up timber engineering with mainstream advances in continuum and computational mechanics. The result is a comprehensive stress analysis regime that enables accurate design and strength predictions of round timber structures.

The LPSA bridge technology, demonstrated in this paper, is in itself a test case of a larger concept of sustainable science (SS), specialized here to its sustainable engineering version. SS is a framework for all technological innovations defined by the following requisite: sustainability, conservation and optimum recycling of natural resources. In practice, the "SS requisite" translates into profitable sustainability. This is because profitable economic activity is generated when commercial value is added to, or created for, natural resources. Therefore, SS innovations could reverse into profitability the heavy tax on social and economic development required to rectify current environmental ills of the Planet.

\section{A Sustainable Engineering Material}

\section{Small Diameter Timber versus Sawn Timber}

Sawn timber is the weakened end product of slicing through a mature tree trunk. Sawing destroys the tough, fibrous and ordered growthring structure of the trunk, resulting 
in a near-random material. Therefore, SDT, with its internal structure intact, is likely to be physically stronger than its sawn equivalent. This is supported by actual tests. ${ }^{3,4}$ It leads to an important and useful conclusion with a built-in factor of safety. In the absence of specific strength data, and until better testing methods are developed for SDT, it is reasonable to use documented values for sawn timber of the same species. For the SDT of special interest here, black locust, the maximum compressive strength (parallel to the grain) reported for sawn wood is 70,2 Mpa. ${ }^{5}$ This is more than 2,5 times the highest compressive strength category specified for structural concrete in highway bridges. ${ }^{6}$

\section{The Jointing Problem}

The value of SDT as a structural material has long been recognized. ${ }^{3,4,7}$ However, it has not been widely or gainfully used due to lack of satisfactory jointing methods. All existing methods are variations of the same carpentry procedure. The ends of a SDT post are slotted and drilled. A metal implant is then embedded and secured, mostly with bolts or wire lacings. Most recently, a European-funded cooperative project ${ }^{3}$ adopted a laced variation of these "embedded joints", developed by its Dutch participant. ${ }^{7}$

Although jointing costs are not considered in Refs. [3] and [7], mass production of the embedded joints is impossible. Some skilled operations and individual attention to each member is needed. This rules out any large-scale, profitable commercial applications of SDT.

In all these joints, the crack-prone SDT fiber is disrupted and exposed to indefinable load concentrations. Engineering modeling and quantification of the state of stress or overall joint strength is impossible. Claimed statistical joint strength estimates, based on laboratory testing, have no bearing on the behavior of actual structures over time. Consequently, no proper structural analysis and strength prediction of any resulting structures is possible beyond simple linear theory diluted by the uncertainties of the embedded joints. More information about current difficulties with SDT and its excellent characteristics under sustained loading appear in Ref. [8].

\section{Resolving the Jointing Problem}

In the LPSA system, the current jointing problem is simply eliminated.
Except for rounding over a short length at each end of the member to slide-fit it into a pipe socket, the SDT section is not compromised. By default, joint strength is at least the strength of the SDT section. Mass production is possible because no skilled operations on individual members are needed. Commercial feasibility of SDT processing is assured.

Once jointing ceases to be a problem, it becomes possible to apply more accurate methods of mathematical and computational modeling and analysis to SDT structures. Combining sustainability and mass processing advantages, all technical, environmental and commercial barriers against using a natural form of wood in the construction of high quality reliable structures like bridges are removed.

\section{Light Prestressed Segmented Arch System}

\section{A Brief Description}

The basic physical aspects of the LPSA system are covered in the author's British Patent GB2206365. A representative two-dimensional LPSA frame would consist of a series of segments made up of single or multiple elongate members with ends slide-fitting into sockets located on metal connectors. Uniform, flat seating is provided for segment member ends inside the sockets for even contact. When a suitable tensioning system is applied, the frame is transformed into a rigid loadbearing structure with unique features, capabilities and flexibilities, especially when SDT posts are used as segment members.

Tensioning produces a structure under a general state of stable compression in the segments. Force equilibrium analyses first help to define a suitable tensioning regime. Methods of continuum and computational mechanics are then used to analyze the elastic-plastic large deformation state of stress and displacement in the connectors and to predict ultimate strength and performance of the total structure. No such facility exists at present for timber structures of any kind.

\section{Unique Features}

The SDT/socket slide-fit mechanism gives rise to an important property: harmless distortion energy dissipation via local SDT rigid-body displacements within the sockets. Therefore, all LPSA structures possess a natural resistance to dynamic actions caused by earthquakes, hurricanes and flooding.

The modular kit form of LPSA structures results in considerable scale - and hence cost - reductions in the manufacture, transportation and assembly. An added advantage over all conventional building methods is disassembly for partial or total reuse of components. It is also possible to replace most segment members and tensioning elements while the structure is in service and to change, within limits, the dynamic characteristics of a structure by redesigning the tensioning system. In regions of dynamic disturbances, structural resilience is very important. Hence, less tensioning is advised.

For SDT components, the only processing needed to transform a small tree standing in a woodland into a structural component in a bridge is cutting accurately to length and rounding the ends to a specific diameter - unless total length rounding is required for formal visual reasons. No drying or seasoning is needed. With black locust SDT and other decay-resistant species, the need for unhealthy preservative treatment does not arise.

So far, connectors used have been simple thin-wall mild steel weldedpipe clusters, hot-dip galvanized for durability. For larger scale standardized structures, cast aluminum alloys or similar materials may result in even more savings, less maintenance, longer life-span and better aesthetics.

Without the large dead loads as in concrete structures, the amount of tensioning needed in LPSA structures is substantially reduced and methods of applying it are simplified. For the same reason, foundations costs are reduced.

\section{LPSA "Schools' Bridges"}

\section{Environmental, Educational and Community Elements}

Funded by a cooperative grant from the Wood In Transportation program, this institute designed and built two identical LPSA bridges, each spanning 19,5 m. Both bridges are located in public parks adjacent to local school campuses. One of them connects the campus to the school's "environmental patch" across a creek. In addition to its main purpose as a sustainable technology demonstration, the project was additionally planned as an exercise in 
environmental education by having school children actively participate in bridge assemblies.

To emphasize the importance of community involvement in environmental innovation, a community alliance was formed to support the project. It consisted of a city government, three local schools, a state department of natural resources and a small "green" manufacturing business that the project helped to start, located on a small family farm.

\section{The LPSA Bridge Structure}

Bridge structure consists of two arched Warren trusses sitting at the ends in saddle support units that also serve as tensioning anchors. These in turn are bolted down to prefabricated units underneath, which are concreted into bridge foundations after it is lifted into final position. For the safety of school children, bridges were first assembled on flat ground. The floor arch was first assembled, Fig. 1. Discarded shipping pallets were collected and used as temporary supports until bridges were tensioned. In the spirit of environmental awareness, a local construction materials business took over the pallets afterward for further reuse.

The trusses are transversely connected via fifteen 3,6 m long, 150+ $\mathrm{mm}$ diameter rounded cross beams supporting the deck units. The result is a formidable arched U-shaped section. Truss segment members are $120 \mathrm{~mm}$ long SDT posts rounded only at the ends to a diameter of $75+\mathrm{mm}$. Otherwise, the natural form of the post is left intact. Even the bark is not removed in order to delay cracking. SDT members at the top are slightly longer than $120 \mathrm{~mm}$ because of the arched geometry. Local school children assembled deck units
(Fig. 2) then segments and connectors of the two trusses (Figs. 3 and 4). A total of 330 SDT posts were used per bridge. No wood drying was needed. All wood used in the trusses, cross beams and deck was black locust.

A truss tensioning system consists of eight $8,6 \mathrm{~mm}$ nominal diameter threewire high-tensile, low-creep strands with a breaking strength of $64,5 \mathrm{kN}$, as used in prestressed concrete work. Polymer-coated by special order, they are "stitched" through truss cells in such a way that all SDT members are in a state of low-to-medium compression. The tensioning system was purposely over-designed for possible future use as a highway bridge test bed. Also, with all strands used in pairs, it is possible to replace the entire system without disturbing the structure.

Once tensioning is completed, a vehicle is driven up the bridge while it is still

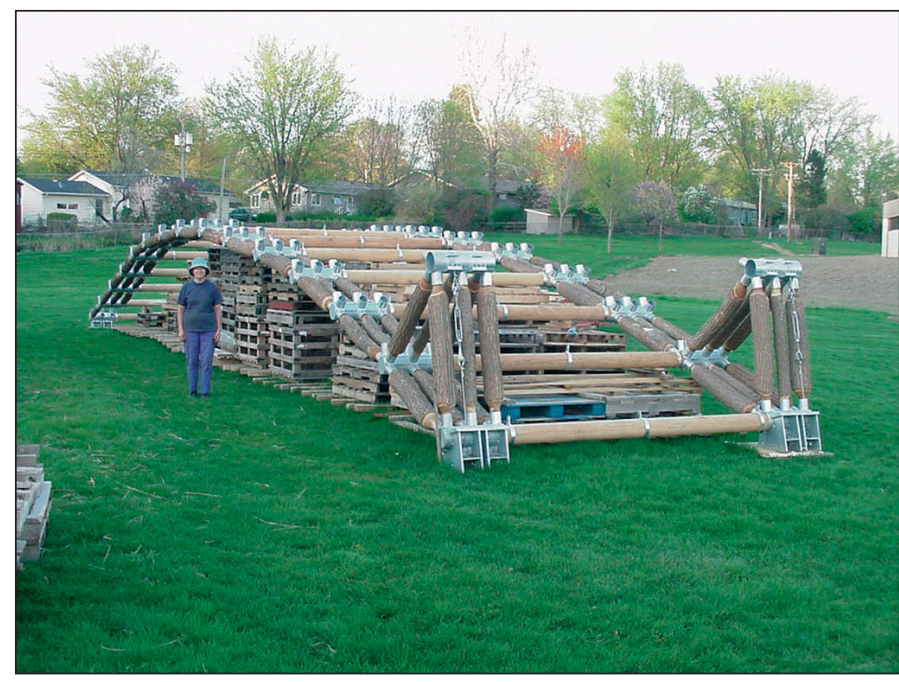

Fig. 1: Floor arch is first assembled on flat ground; discarded shipping pallets are used here as temporary supports

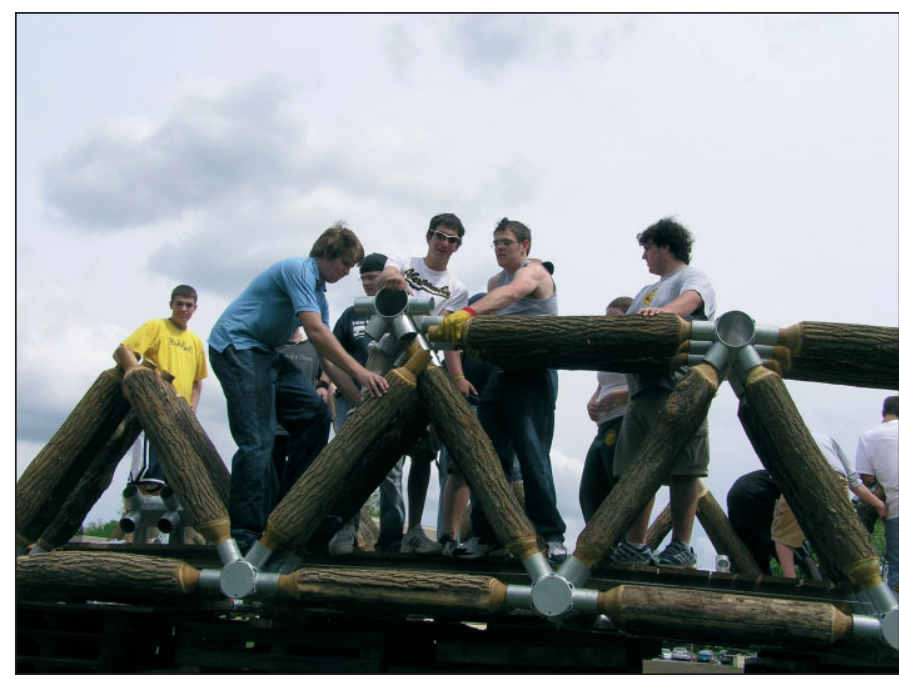

Fig. 3: Students assemble segment members (SDT posts) and connectors

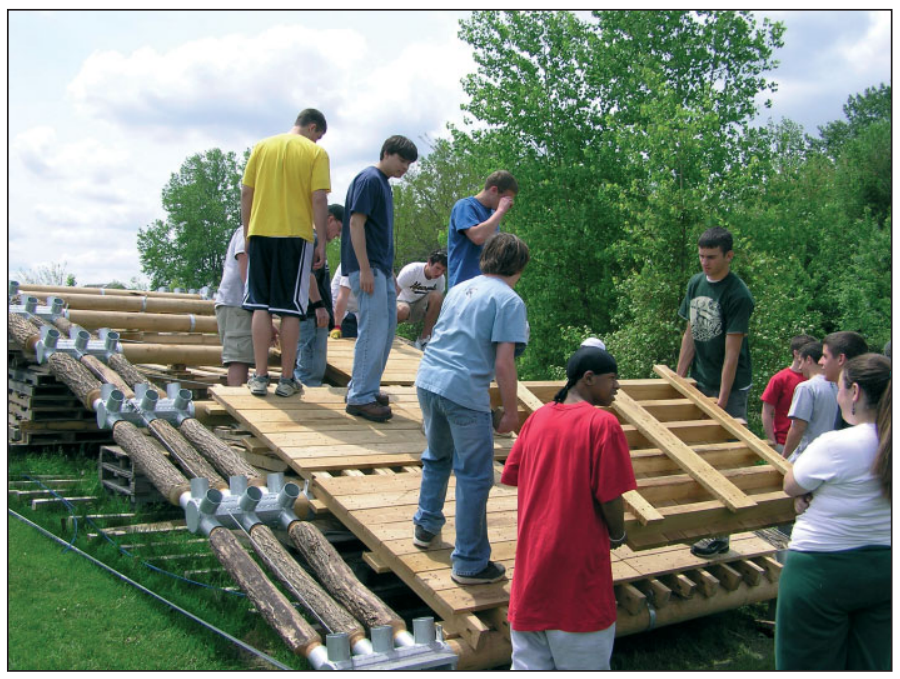

Fig. 2: High school students assemble deck units; these will later be flexibly anchored to the cross beams under them

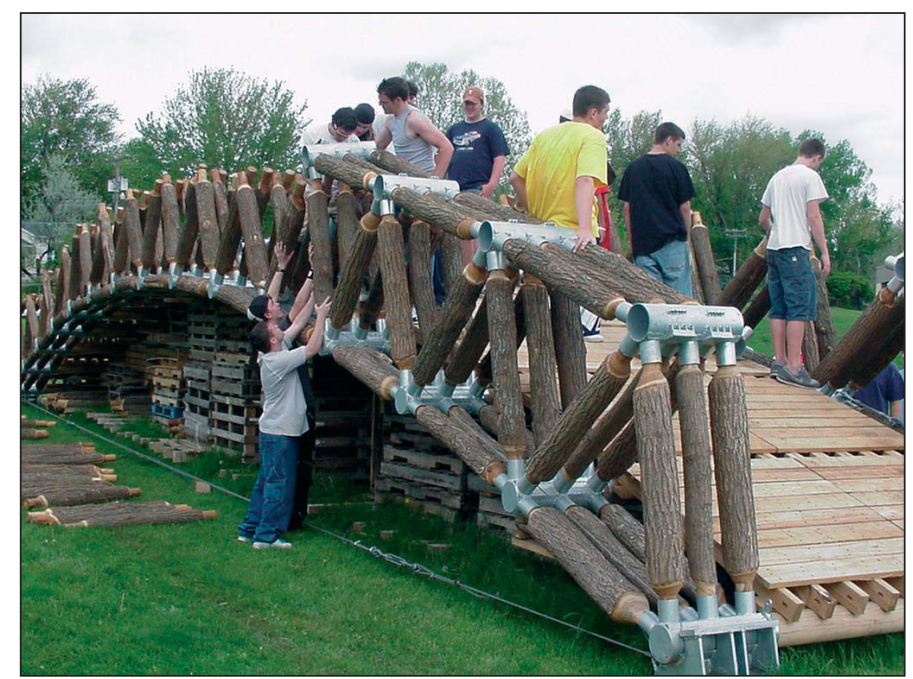

Fig. 4: One of two bridge trusses is nearing completion, being assembled from one end to the other 
sitting directly on the ground, Fig. 5. Figure 6 is a view of bridge underside showing under-floor bracing strands and deck units interlocking over cross beams. Figure 7 depicts a tensioning anchor unit. The saddle seating of a truss end approximates a pin joint. The eight gripped tensioning strands are also shown prior to trimming. Figure 8 is a solid model close-up showing the tensioning strands' "stitching" pattern. The actual blue strands are colored red in the model for clarity.

Tensioning is applied via a simple mechanical procedure. Tensioning grips, underneath plate washers, are applied at strand ends. Threaded bolts through the top plate of the anchor unit push down against the washers, imparting tension to the strands. Galvanized metal packings are used on top of the washers before the threaded bolts are withdrawn. An auxiliary grip is installed at the next connector to facilitate strand tensioning, de-tensioning and replacement.

Live loads used in the initial finite element analysis followed the American Association of State Highway and Transportation Officials (AASHTO's) recommended values for pedestrian bridges. $^{9}$ This produced low values of stresses, deflections and lateral thrusts. Several numerical loading experiments were then conducted with various loads and patterns. A final run simulated AASHTO's H-20 highway bridge loading, ${ }^{6}$ assuming a 20 -t $(178 \mathrm{kN})$ vehicle, with $80 \%$ of the load applied at the center of the bridge. Even with a highly exaggerated strand tension of $27 \mathrm{kN}$, the results barely registered localized plasticity in the central bridge connector. In reality, only nominal tension (at least an order of magnitude less) was applied to the strands, producing a sturdy, rigid structure with built-in flexibility.

An initial loading test to demonstrate the load carrying capacity predicted by LPSA analysis was performed by parking three vehicles on the bridge, Figs. 9 and 10. Experience gained from both actual and numerical loading tests indicates that these "pedestrian" bridges are capable of carrying highway bridge loads. However, this particular geometry needs to be modified for such applications. Bridge deck would be carried by the top connectors and a suitable railing system be added. Curvature would be reduced or eliminated. The U-shaped bridge crosssection would then become an even more formidable box section or multiple box sections. LPSA versions of

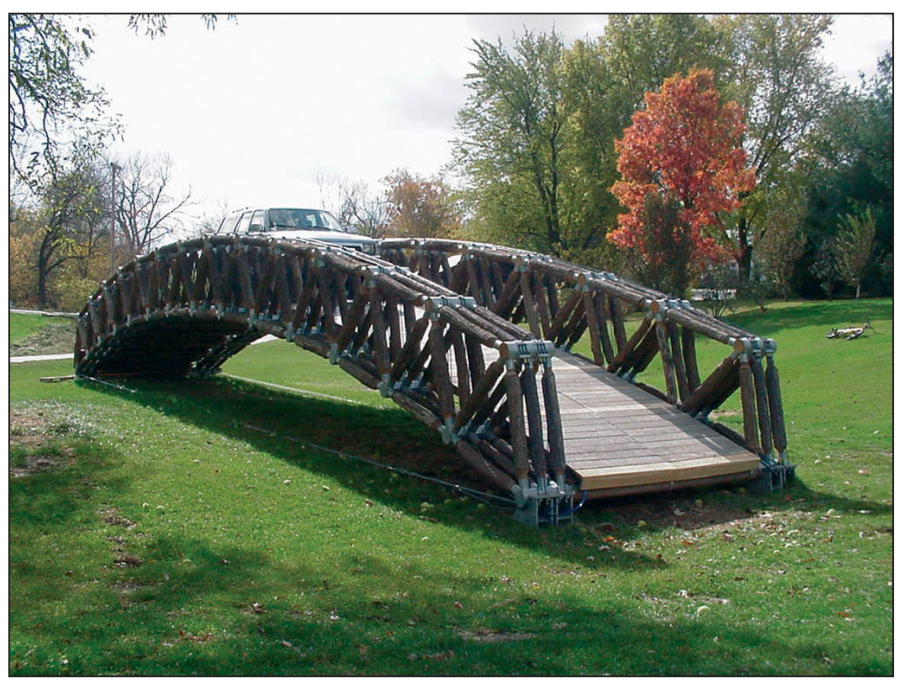

Fig. 5: Bridge is completed and tensioned; with ends still tied to prevent arch spreading, a vehicle is driven up on top

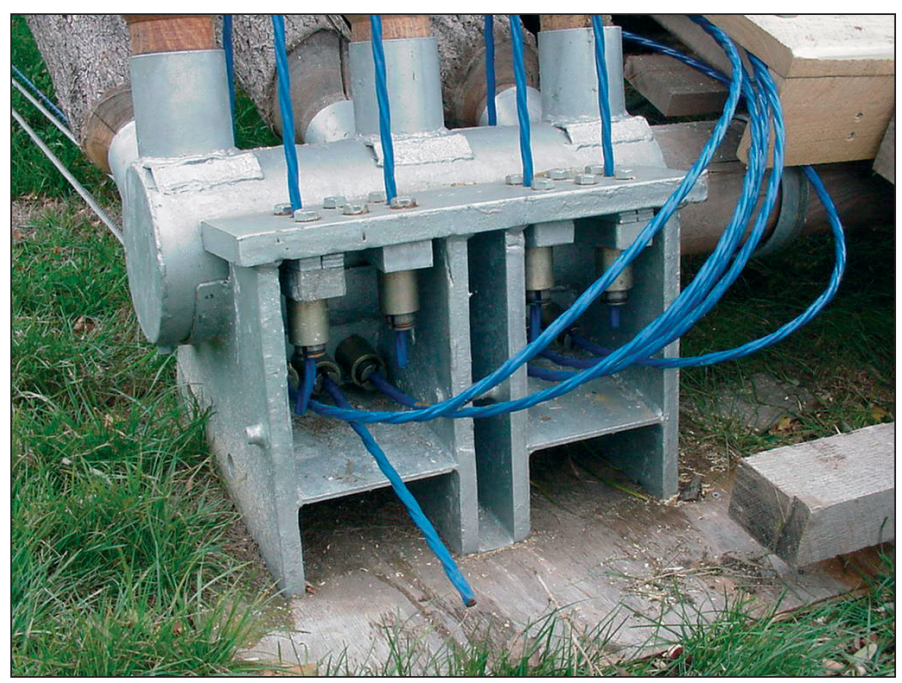

Fig. 7: Tensioning anchor unit showing "saddle" seating of truss end and strand anchor assemblies

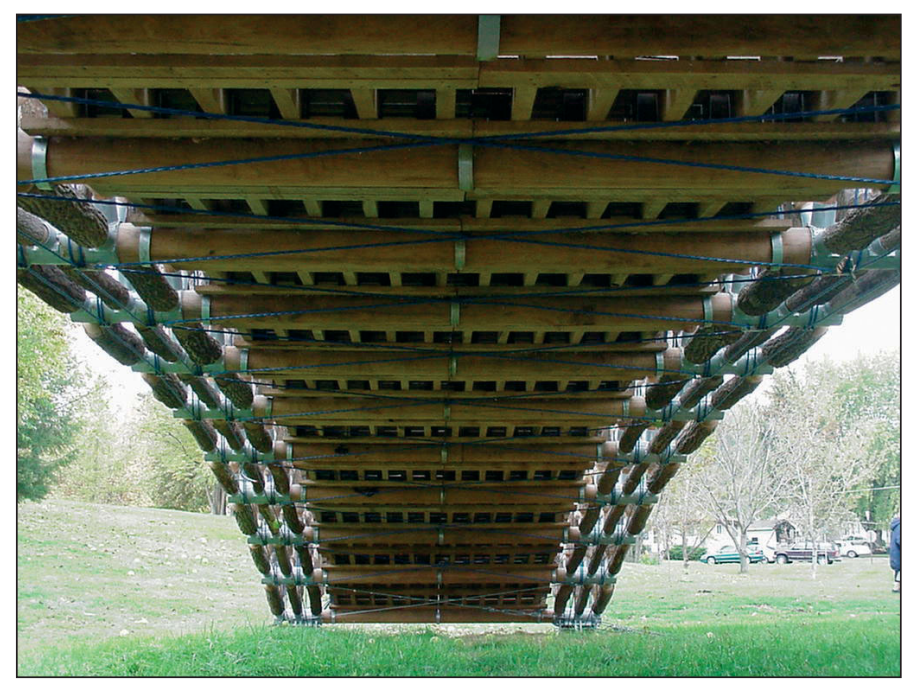

Fig. 6: A view of bridge underside depicting cross-bracing strands and the interlocking deck units

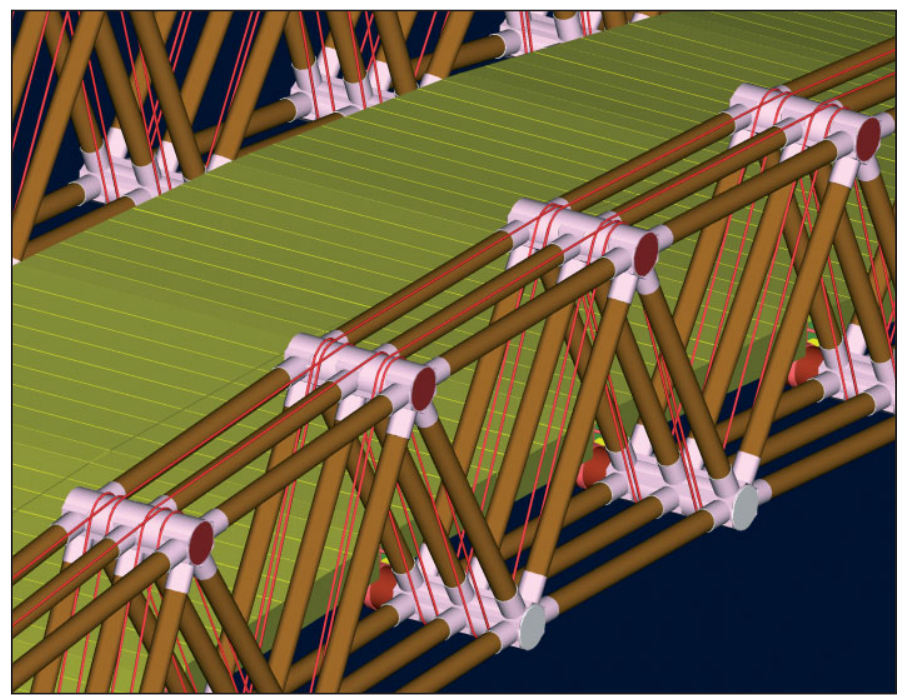

Fig. 8: A solid model close-up of bridge depicting the eight-strand truss tensioning arrangement (in red). Actual strands are blue (see Fig. 7) 


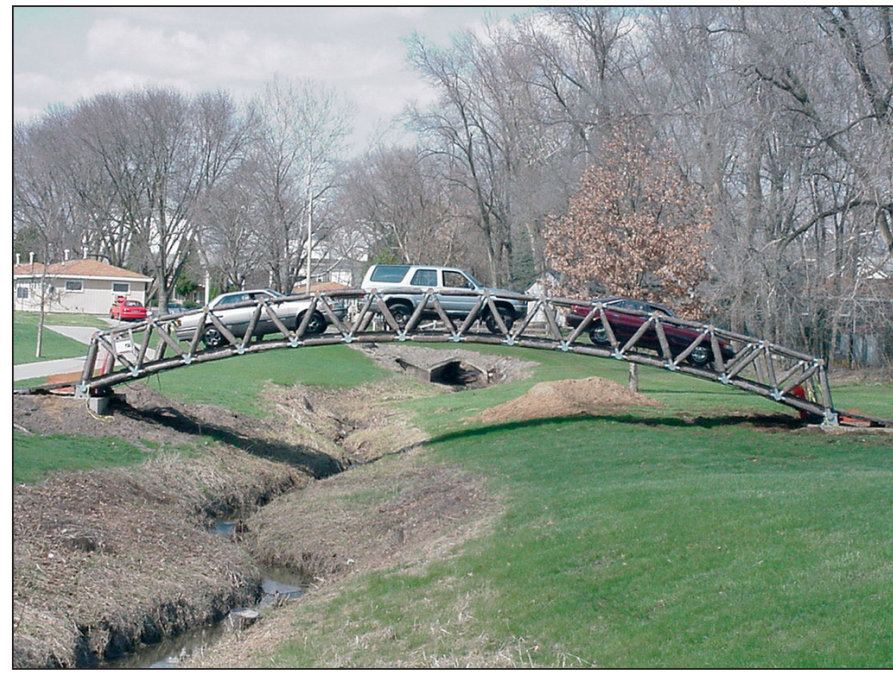

Fig. 9: Bridge sitting on permanent foundations with three vehicles parked on it

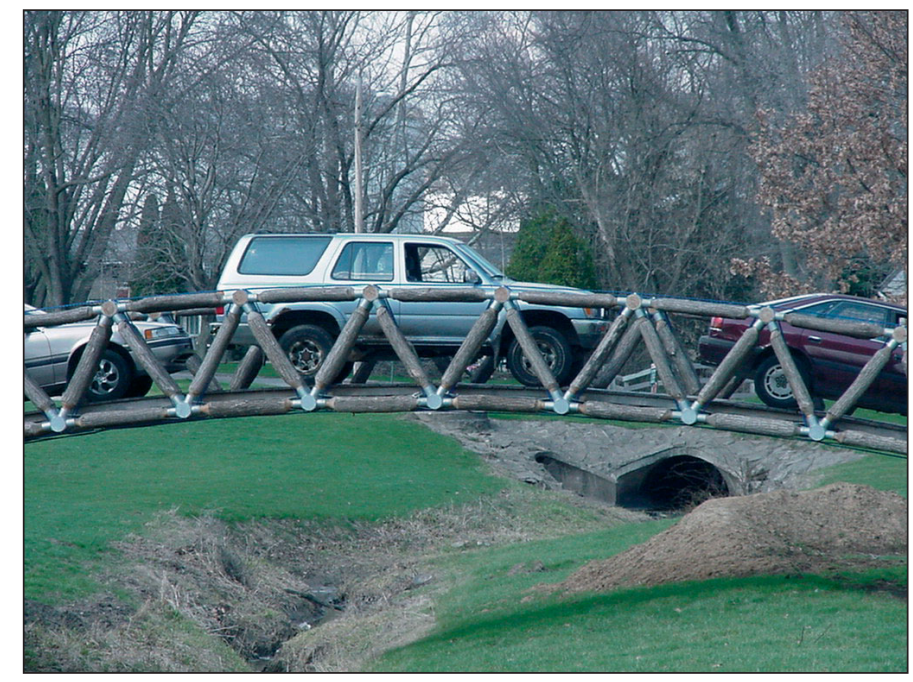

Fig. 10: A close-up of bridge in Fig. 9; vehicle loading is an early indication of the highway-bridge-grade capacity of this "pedestrian" bridge as predicted by analysis all existing bridge configurations, and more, can be readily developed.

\section{LPSA Highway Bridge Initiative}

The environmental, economic and technical merits of LPSA bridges as discussed above, point to a promising "green" highway bridge technology whose time is overdue. This nonprofit engineering research institute and public charity invites interested parties around the world to cooperate with it on further intellectual development and commercialization of the technology. A good starting activity for this initiative could be to carry out extensive loading tests on the two bridges above to verify the analytical development so far.

\section{Conclusion}

By resolving the jointing problem, the LPSA structural system enables lowcost, large-scale utilization of SDT as a premium structural material and a better alternative to mature structural sawn timber. For the first time, methods of continuum and computational mechanics are applied in the stress analysis of timber structures. Creating commercial value and applications for SDT could develop into profitable ways of saving what is left of the world's forests and for sustainable development. The LPSA is a demonstration of the validity of a larger concept of sustainable science and engineering, requiring sustainability, conservation and optimum recycling of natural resources. This could tap a new field of research and innovation with sustainable solutions to global problems like deforestation, forest fires, poverty and durable shelter. A promising sustainable, low-cost, durable LPSA highway bridge technology is proposed. Potential worldwide partners are invited to participate in its development.

\section{Acknowledgement}

The bridges project described in this paper was funded by a cooperative grant from the Wood In Transportation program.

\section{References}

[1] Upkeep Costs Rise as USA's Bridges Age, USA Today, 20 October, 2006, 3A (newspaper report).
[2] NASH G. Wooden Fences. Taunton Press, Newtown, CT, USA, 1999; 96.

[3] Ranta-Maunus A (ed). Round SmallDiameter Timber for Construction. Final Report of Project EU FAIR CT 95-0091, Technical Research Centre of Finland (VTT), Finland, 1999.

[4] Darby HJ. Building with home grown round timber. Farm Build. Eng. 1987; 3(3): 18-21.

[5] Wood Handbook: Wood as an Engineering Material. Forest Products Society: USA, 1999; 4-10.

[6] Standard Specifications for Highway Bridges. AASHTO (American Association of State Highway and Transportation Officials): Washington, DC, USA, 2002.

[7] Huybers P. Space structures of timber poles. In Shells, Membranes and Space Frames; Proceedings, IASS Symposium, Vol. 3, Heki K (ed), Osaka, Elsevier Science Publishers B. V., Amsterdam, The Netherlands, 1986; 293-300.

[8] Al-Khattat I. The LPSA: an inevitable sustainable technology. Innovative Wooden Structures and Bridges, Proceedings, IABSE Conference, Lahti, Finland, 2001; 379-384.

[9] Guide Specifications for Design of Pedestrian Bridges. AASHTO (American Association of State Highway and Transportation Officials): Washington, DC, USA, 1997. 